\title{
The Effect and Compensation of Phase Noise on Orthogonal Frequency Division Multiplexing (OFDM) System
}

\author{
Yubin Lin \\ Greater Atlanta Christian School, Norcross, Georgia, USA \\ Email: linyubin1999@gmail.com
}

How to cite this paper: Lin, Y.B. (2018) The Effect and Compensation of Phase Noise on Orthogonal Frequency Division Multiplexing (OFDM) System. Circuits and Systems, 9, 107-115.

https://doi.org/10.4236/cs.2018.97011

Received: December 8, 2017

Accepted: July 27, 2018

Published: July 30, 2018

Copyright $\odot 2018$ by author and Scientific Research Publishing Inc. This work is licensed under the Creative Commons Attribution International License (CC BY 4.0).

http://creativecommons.org/licenses/by/4.0/

\section{(c) (i) Open Access}

\begin{abstract}
Orthogonal Frequency Division Multiplexing (OFDM) is characterized by its high data rate. However, the modulation method used in the system is subject to the influence of phase noise due to the need of time synchronization. In this paper, an algorithm based on MMSE (minimum mean square error) is developed to compensate the influence of both the common phase error (CPE) and inter carrier interference (ICI), which are two aspects of phase noise, under common Gaussian white noise. The result of noise cancellation is presented in signal-to-noise ratio (SNR) and symbol error rate (SER). Like digital signal in general, SNR can reduce SER with or without phase noise compensation. The compensation of phase noise significantly reduces the SER of the decoded signal. However, the bandwidth of phase noise still determines the signal accuracy. Under high bandwidth of phase noise, increasing SNR will only slightly increase SER, which is not efficient.
\end{abstract}

\section{Keywords}

Orthogonal Frequency Division Multiplexing, Phase Noise, Mean Square Error

\section{Introduction}

Orthogonal Frequency Division Multiplexing (OFDM) is widely used in the telecommunication industry. First introduced in 1960s, OFDM has wide application in data transmission, including radio and HDTV signal [1] [2] as well as the standard for WLAN system, which includes the widely used Wi-Fi [3]. The high bandwidth of OFDM signal gives its resistance to narrowband interference, high data rate [4] thus high spectral efficiency [5]. However, OFDM signal quality can 
be easily degraded by some conditions such as carrier frequency offsets, sampling clock offsets and low frequency phase noise [6]. The phase noise problem is critical to the system's efficiency because phase noise produces inter carrier interference (ICI) and the result rotation of the constellation map is called common phase error (CPE) which degrades the quality of the receiving signal [7].

Phase noise may be introduced to the system in many steps. The bandwidth of the practical oscillator can add phase noise to the signal thus compromising the orthogonality of the signal, the same "unclean" oscillator can also produce phase noise when demodulating the incoming OFDM signal [8]. Phase noise has great influence on the signal quality of OFDM in HDTV and other situations where high data rate is required because in these applications [9], 64-QAM modulation is often used in order to meet the data rate requirement [10] and this modulation is sensible to phase noise [9]. In addition, phase noise can affect time synchronization of OFDM signals, compromising the accuracy of time signal such as that used on GPS. Thus, the problem of phase noise in OFDM needs to be addressed and solved due to its wide application. Many have done research into this problem by getting a better approximation of phase noise using phase-locked-loop (PLL) [11] and other advanced estimation algorithms [12].

In this experiment, the phase noise is produced through Wiener Process-a stochastic process with random increments at non-negative time $t$ [13]. This is an appropriate simulation of phase noise [14]. Under this process we analyze the effect phase noise has on the OFDM signal as well as how to suppress the influence of phase noise through channel estimation [15].

The paper begins with introduction of the OFDM system used in this project, and then a phase noise introduced into the receiver. An algorithm is used to correct the effect of phase noise, and the results of the correction are displayed as symbol error rate versus signal to noise ratio.

\section{OFDM System}

OFDM signal is generated from 64 quadrature-amplitude modulation (QAM) under inverse fast Fourier transform (IFFT). In order to avoid inter-carrier interference, cyclic prefix, a repetition of last couple of symbol appended to the start of the signal, is used to preserve orthogonality. Despite the loss of bandwidth and power, cyclic prefix significantly improves the signal quality and is used very commonly in modern OFDM system. If the length of the cyclic prefix is $\mathrm{L}$, and the original signal has $\mathrm{N}$ symbols, the final transmission will have $(\mathrm{N}+$ L) symbols [16]. In addition, several pilot signals are added to replace some signal. This is for the channel estimation in the receiving the signal and will be further explained later. Finally, the signal goes through a parallel to serial converter in order to be sent one at a time in time domain (Figure 1).

During the transmission, additive white Gaussian noise (AWGN) with different signal to noise ratio (SNR) as well as phase noise is added to simulate the 


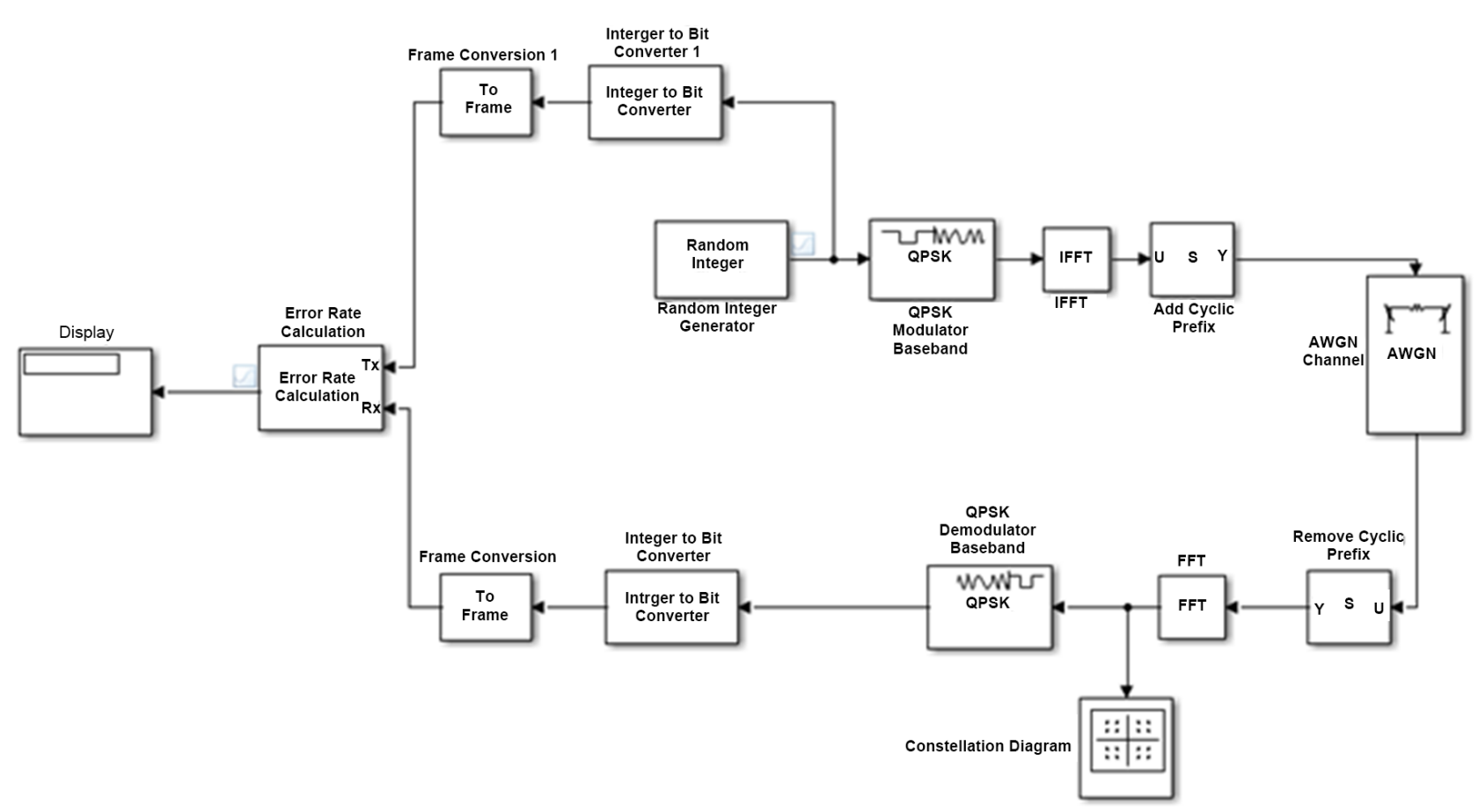

Figure 1. A comprehensive block diagram of OFDM system.

path [17]. At the receiving end, signal is converted from serial to parallel in order to separate each subcarrier, then the cyclic prefix of each of the subcarrier is removed. The pilots in incoming signal are used for channel estimation due to its known given value. Finally, the signal goes through fast Fourier transform (FFT) and is demodulated to the original data set. The demodulated data set is then compared with the original data set, thus enabling to calculate Bit error rate (BER) and symbol error rate (SER).

\section{Phase Noise}

Without phase noise, the receive signal can be presented as:

$$
r(n)=(y(n) h(n))+\xi(n)
$$

where $r(n)$ denotes the received nth subcarrier OFDM signal, $y(n)$ for modulated nth subcarrier signal, $h(n)$ for the channel impulse response of nth subcarrier signal and for the AWGN of nth subcarrier signal. The symbol stands for convolution.

The phase noise poses two problems that can significantly reduce the efficiency of the system: Common phase error (CPE) and Inter carrier Interference (ICI). The modulated signal amplitude with phase noise for each carrier can be written as:

$$
r(n)=(y(n) \otimes h(n))+\mathrm{e}^{j \phi(n)}+\xi(n)
$$

The term represents phase noise at the receiving oscillator. The phase noise is generated by wiener process in order to get Brownian motion. The wiener 
process can be written as

$$
\phi(n+1)=\phi(n)+w(n)
$$

where $w(n)$ represents gaussian random variable with mean of zero and standard deviation of $4 \pi \Delta f 3_{d B} T_{s}$ where denotes the $3 \mathrm{~dB}$ bandwidth of the phase noise and Ts denotes the sampling instant. The wiener phase noise in time domain $(\phi(n))$ with mean of zero looks like (Figure 2):

After removing cyclic prefix and taking the Discrete Fourier Transform (DFT) the received signal in frequency domain then becomes:

$$
R_{m, l}=X_{m, l} H_{m, l} I_{m}(0)+\sum_{n=0, n \neq l}^{n-1} X_{m n} H_{m n} I_{m}(l-n)+\eta_{m, l}
$$

denotes the received signal at $m$ th symbol and $l$ th subcarrier $(l=0,1, \cdots, n-1)$. denotes the AWGN after DFT. The term $\operatorname{Im}(i)$ denotes the DFT of during one symbol and this term is the representation of CPE, which results the rotation of the constellation diagram:

$$
I_{m}(i)=1 / n \times \sum_{n=0}^{n-1} \mathrm{e}^{-j 2 \pi n i / n} \mathrm{e}^{j \phi(n)}
$$

The term

$$
\sum_{n=0, n \neq l}^{n-1} X_{m n} H_{m n} I_{m}(l-n)
$$

denotes ICI.

\section{Common Phase Error}

The phase noise is constructed by two parts: Common Phase Error (CPE) and Inter carrier Interference (ICI). CPE is caused by the rotation of the phase in a specific subcarrier only, while ICI is caused by the interference between subcarriers around a specific subcarrier. The phase noise compromises the orthogonality of the incoming signal, resulting overlaps between carriers. Common Phase Error can be easily corrected by the comparison between pilot signal and the incoming signal. Least Square method compares the received pilot signal with the known pilot value, and uses this ratio to correct the rest of the channel. Due to the unknown nature of channel condition, multiple subcarriers are designed specifically to transmit pilot signal for the more accurate estimation of a portion of the signal. Pilot value can be expressed as $\mathrm{T}$, and the subcarrier that contains pilot value is $\mathrm{i}$ and the result (the ratio) is expressed $\mathrm{E}(i)$. The LS method of estimation can be written as:

$$
E(i)=r(i) / T
$$

The ratio can then be used to compensate the channel condition:

$$
r(n)^{\prime}=r(n) / E(i)
$$

Here's the transmitted and received signal (without phase noise) with LS channel (Figure 3).

In addition to LS, MMSE (Minimum Mean Square Error) is also used in this type of compensation. The formula for MMSE can be written as: 


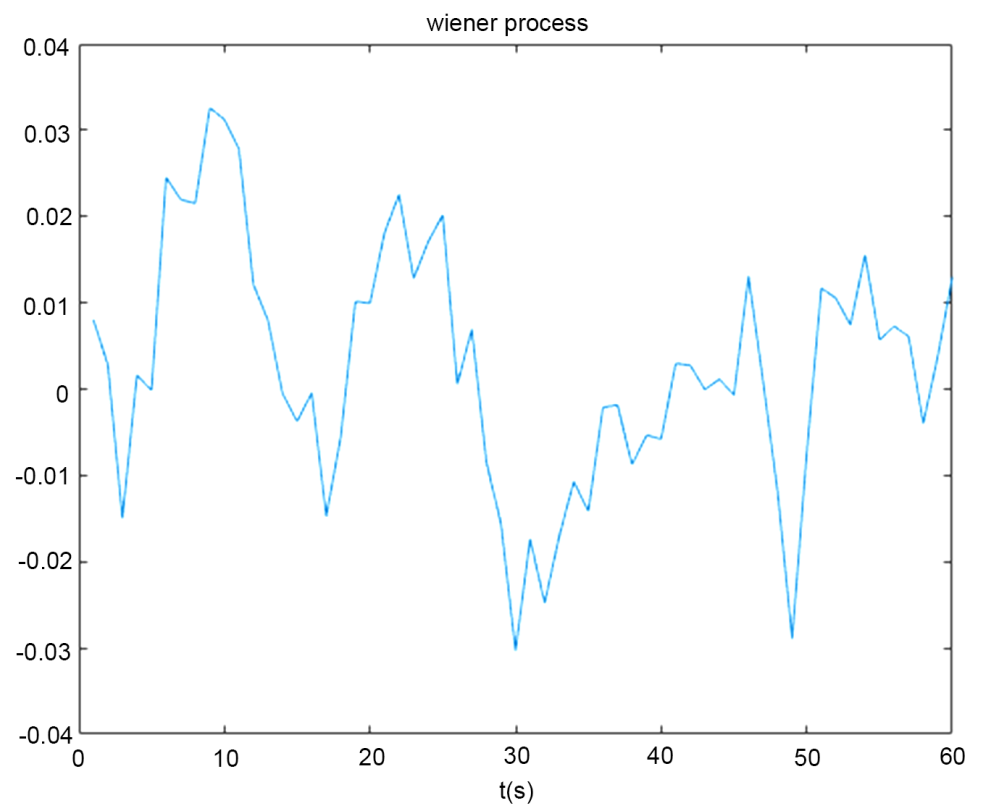

Figure 2. Wiener Process in respect with time.


Figure 3. Transmitted and received signal without any phase noise.

$$
I(m)=\sum R(k) X(k) \underline{H}(k) / \sum|X(k) \underline{H}(k)|^{2}
$$

where $\underline{H}(k)$ is the signal pre-estimation at $k$ th subcarrier.

\section{Inter-Carrier Interference}

Besides CPE, ICI needs to be addressed as well. From the previous formula

$$
r(n)=(y(n) \otimes h(n)) \mathrm{e}^{j \phi(n)}+\xi(n)
$$


We know that by multiplying the received signal with the $\mathrm{e}^{-j \phi(n)}$, conjugate of $\mathrm{e}^{j \phi(n)}$, the phase noise would cancel out. However, this method can only be applied in the receiver after the transformation from time to frequency domain; thus the multiplication becomes convolution for the removal of the phase noise. And because this compensation is done at the frequency domain, a discrete Fourier transform for all conjugate is required. Let Um denotes the phase noise factor after DFT in $m^{\text {th }}$ subcarrier: $U m=D F T\left\{\mathrm{e}^{-j \phi(n)}\right\}$. Thus, circularly convolving the received signal with Um could compensate the phase noise.

However, in real life, simply acquiring known value of phase noise is impossible. Thus, estimation phase noise is essential to the compensation. First, a standard demodulation is performed in the incoming OFDM signal. Then using LS or other signal estimation to derotate the constellation graph in order to get eliminate CPE. By the degree of derotation, a rough estimation of phase noise can be estimated. The order of estimation determines how many interactions between the center subcarrier and adjacent subcarriers. While it is possible to estimate all the order phase noise (to find out the interference between every sets of subcarriers), it would require huge calculation power and reduce the speed of decoding the signal, thus impractical for actual use. However, thirds order can give relatively accurate estimation as shown in the graph below (Figure 4):

Third order creates a seven by seven matrix. The diagonal of this matrix will be the estimation. By filling up the rest of the matrix zero, we can use this matrix to circular convolve the received signal to give an efficient estimation. The estimated received signal can be written as:

$$
R(m)=A_{m} J_{m}+\varepsilon_{m}
$$

where $A_{m}=\left[A_{l_{1}}+\cdots+A_{l_{k}}\right]$ and $A_{l}=\left[H_{m, l} X_{m, l}+\cdots+H_{m, l+u} X_{m, l+u}\right]$, in addition,

$$
J_{m}=\left[I_{m(0)}+\cdots+I_{m(u)}\right] .
$$

The vector A and J represents the ICI phase noise in the equation

$$
\sum_{n=0, n \neq l}^{n-1} X_{m n} H_{m n} I_{m}(l-n) .
$$

To address the term $\varepsilon_{m}$, AWGN and power of phase noise in time domain is added.

$$
J_{m}=M R_{m}
$$

where, $M=R_{J_{m} J_{m}} A_{m}^{H}\left(A_{m} R_{J_{m} J_{m}} A_{m}^{H}+R_{\varepsilon_{m} \varepsilon_{m}}\right)^{-1}, R_{J_{m} J_{m}}$ and $R_{\varepsilon_{m} \varepsilon_{m}}$ are the correlation matrices of $J_{m}$ and $\varepsilon_{m}$.

With the estimated signal solved, MMSE can be used to estimate the incoming signal.

\section{Results and Discussion}

Figure 5 is the constellation diagram before and after the third-order compensation of phase noise. Although the data on the edge of the derotated diagram 


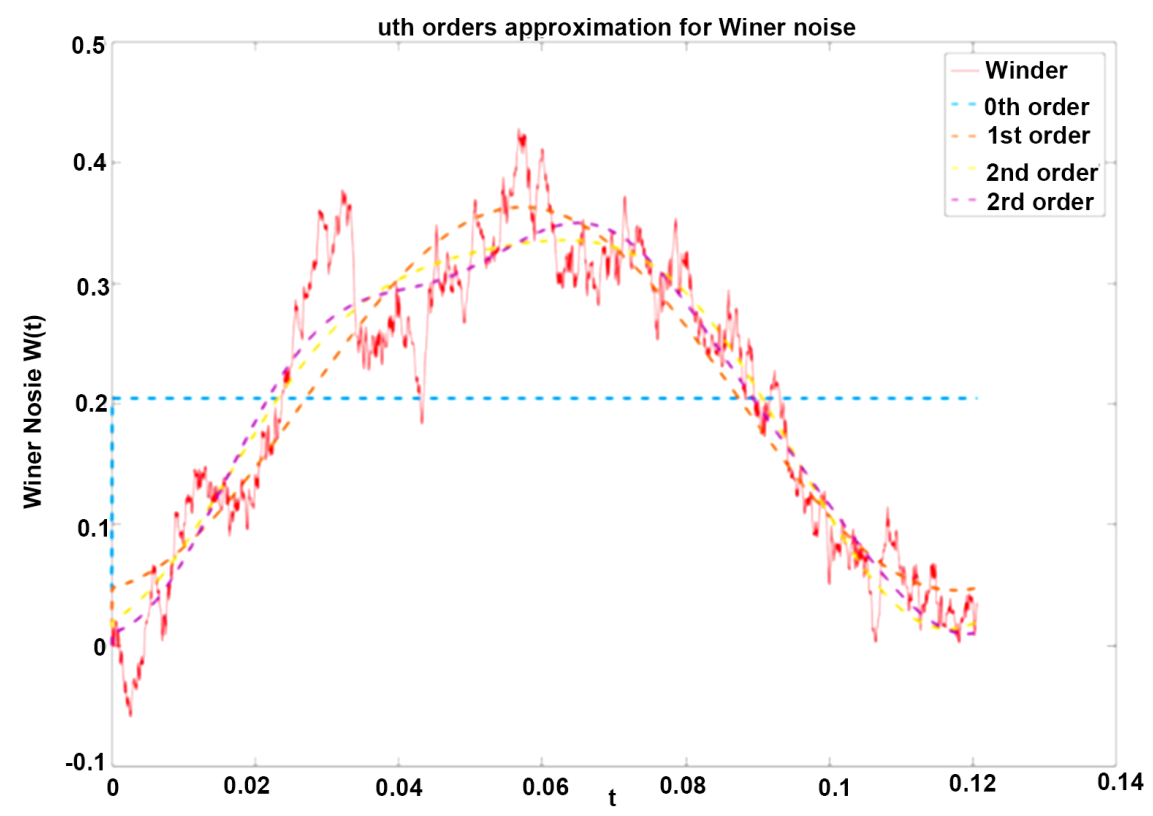

Figure 4. Estimation of the wiener process.
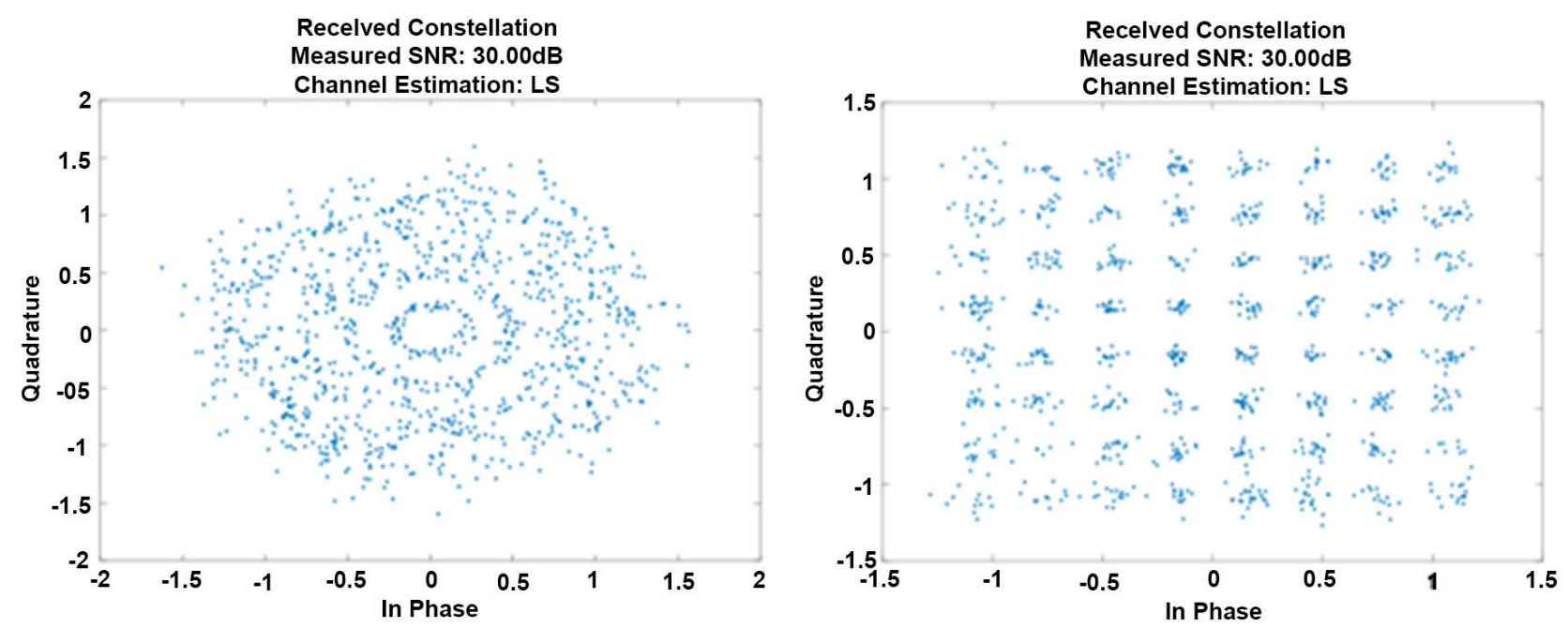

Figure 5. Received signal in constellation plot before and after phase noise compensation.

seems compromised, most of the data can be obtained by compensation CPE and ICI, which is an adequate compromise between data rate and data fidelity.

Under different bandwidths of phase noise, a clear trend is shown in Figure 6. In general, symbol error rate (SER) significantly decreases after the compensation of phase noise under all phase noise bandwidths. Under low signal-to-noise ration conditions, the existence of phase noise, as well as the bandwidth of phase noise, does not pose any discernable changes in signal quality measured by SER. However, we can see the effect of phase noise when SNR is greater than $20 \mathrm{db}$. At $25 \mathrm{~dB}$, the SER without phase noise is about ten times smaller than the one with $150 \mathrm{~Hz}$ of phase noise. Without the presence of phase noise, the signal quality exponentially increases at the rate faster than any other conditions. 

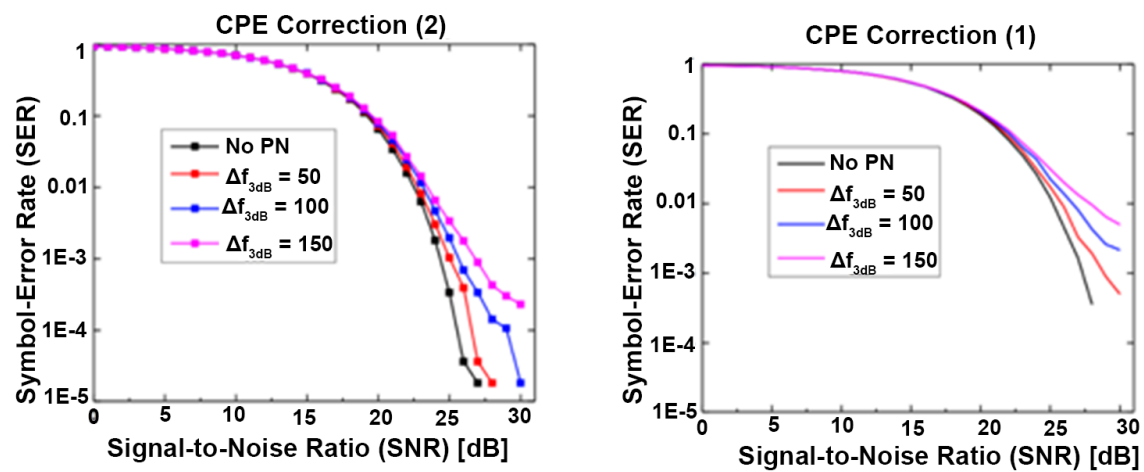

Figure 6. Relationship between SNR and SER under various amount of phase noise.

Under the presence of phase noise, the signal quality increases with the increase of SNR, but less than that without phase noise. With large amount of phase noise present, for example, $=150 \mathrm{~Hz}$, the improvement of signal quality is "flat out" even when the SNR increases. Thus, a high SNR ratio will not result in corresponding improvement on SER under severe phase noise conditions. Increase order of estimation can improve the results but cannot cancel the effect of the phase noise completely. Further research is needed to minimize the effect of phase noise under high SNR conditions.

\section{References}

[1] Zou, W.Y. and Wu, Y.Y. (1995) COFDM: An Overview. IEEE Transactions on Broadcasting, 41, 1-8. https://doi.org/10.1109/11.372015

[2] Weinstein, S.B. (2009) The History of Orthogonal Frequency-Division Multiplexing [History of Communications]. IEEE Communications Magazine, 47, 26-35. https://doi.org/10.1109/MCOM.2009.5307460

[3] Wu, S.P. and Bar-Ness, Y. (2002) A Phase Noise Suppression Algorithm for OFDM-Based WLANs. IEEE Communications Letters, 6, 535-537. https://doi.org/10.1109/LCOMM.2002.806468

[4] Dakshi, A., et al. (1998) Space-Time Coded OFDM for High Data-Rate Wireless Communication over Wideband Channels. VTC98. 48 th IEEE Vehicular Technology Conference, Pathway to Global Wireless Revolution (Cat. No.98CH36151), Ottawa, Ont., 21-21 May 1998, Vol. 3.

[5] Mikael, S., et al. (2003) Attaining Both Coverage and High Spectral Efficiency with Adaptive OFDM Downlinks. 2003 IEEE 58th Vehicular Technology Conference VTC 2003-Fall (IEEE Cat. No.03CH37484), Orlando, FL, 6-9 October 2003, Vol. 4.

[6] Tomba, L. (1998) On the Effect of Wiener Phase Noise in OFDM Systems. IEEE Transactions on Communications, 46, 580-583. https://doi.org/10.1109/26.668721

[7] Zou, Q.Y., Tarighat, A. and Sayed, A.H. (2007) Compensation of Phase Noise in OFDM Wireless Systems. IEEE Transactions on Signal Processing, 55, 5407-5424. https://doi.org/10.1109/TSP.2007.899583

[8] Mourad, M., Hasnaoui, S. and Bouallegue, R. (2007) Analysis of Frequency Offsets and Phase Noise Effects on an OFDM $802.11 \mathrm{~g}$ Transceiver. International Journal of Computer Science and Network Security, 5, 87-91.

[9] Patrick, R. and Kaiser, S. (1995) Analysis of the Effects of Phase-Noise in Ortho- 
gonal Frequency Division Multiplex (OFDM) Systems. Proceedings IEEE International Conference on Communications ICC 95, Seattle, WA, 18-22 June 1995, Vol. 3.

[10] Fazel, K. and Ruf, M.J. (1993) A Hierarchical Digital HDTV Transmission Scheme for Terrestrial Broadcasting. Proceedings of IEEE Global Telecommunications Conference GLOBECOM93, Houston, TX, 29 November-2 December 1993. https://doi.org/10.1109/GLOCOM.1993.318420

[11] Petrovic, D., Rave, W. and Fettweis, G. (2007) Effects of Phase Noise on OFDM Systems with and without PLL: Characterization and Compensation. IEEE Transactions on Communications, 55, 1607-1616. https://doi.org/10.1109/TCOMM.2007.902593

[12] Casas, R.A., Biracree, S.L. and Youtz, A.E. (2002) Time Domain Phase Noise Correction for OFDM Signals. IEEE Transactions on Broadcasting, 48, 230-236. https://doi.org/10.1109/TBC.2002.803711

[13] Denis, P., Rave, W. and Fettweis, G. (2004) Intercarrier Interference Due to Phase Noise in OFDM-Estimation and Suppression. IEEE 60 th Vehicular Technology Conference VTC2004-Fall, Los Angeles, CA, 26-29 September 2004.

[14] Alper, D., Mehrotra, A. and Roychowdhury, J. (2000) Phase Noise in Oscillators: A Unifying Theory and Numerical Methods for Characterization. IEEE Transactions on Circuits and Systems I: Fundamental Theory and Applications, 47, 655-674. https://doi.org/10.1109/81.847872

[15] Van De Beek, J.-J., et al. (1995) On Channel Estimation in OFDM Systems. 1995 IEEE 45th Vehicular Technology Conference Countdown to the Wireless Twenty-First Century, Chicago, IL, 25-28 July 1995, Vol. 2.

[16] Bingham, J.A.C. (1990) Multicarrier Modulation for Data Transmission: An Idea Whose Time Has Come. IEEE Communications Magazine, 28, 5-14. https://doi.org/10.1109/35.54342

[17] Schmidl, T.M. and Cox, D.C. (1997) Robust Frequency and Timing Synchronization for OFDM. IEEE Transactions on Communications, 45, 1613-1621. https://doi.org/10.1109/26.650240 